Published in final edited form as:

Curr Opin Endocrinol Diabetes Obes. 2010 October ; 17(5): 472-477. doi:10.1097/MED.

0b013e32833c5c48.

\title{
Obesity genes and insulin resistance
}

\author{
Anna C. Belkina and Gerald V. Denis \\ Boston Nutrition Obesity Research Center, Cancer Research Center, Boston University School of \\ Medicine, Boston, Massachusetts, USA
}

\section{Abstract}

Purpose of review-The exploding prevalence of insulin resistance and Type 2 diabetes (T2D) linked to obesity has become an alarming public health concern. Worldwide, approximately 171 million people suffer from obesity-induced diabetes and public health authorities expect this situation to deteriorate rapidly. An interesting clinical population of 'metabolically healthy but obese' (MHO) cases is relatively protected from T2D and its associated cardiovascular risk. The molecular basis for this protection is not well understood but is likely to involve reduced inflammatory responses. The inflammatory cells and pathways that respond to overnutrition are the primary subject matter for this review.

Recent findings-The chance discovery of a genetic mutation in the $\mathrm{Brd} 2$ gene, which is located in the class II major histocompatibility complex and makes mice enormously fat but protects them from diabetes, offers revolutionary new insights into the cellular mechanisms that link obesity to insulin resistance and T2D. These Brd2-hypomorphic mice have reduced inflammation in fat that is normally associated with insulin resistance, and resemble MHO patients, suggesting novel therapeutic pathways for obese patients at risk for T2D.

Summary-Deeper understanding of the functional links between genes that control inflammatory responses to diet-induced obesity is crucial to the development of therapies for obese, insulin-resistant patients.

\section{Keywords}

$B r d 2$ gene; inflammation; insulin resistance; metabolically healthy obese; obesity

\section{Introduction}

During the last decade, the medical community has determined that obesity (defined as BMI $\geq 30$ ) due to over-nutrition and reduced physical activity has become not only the most prevalent nutritional disorder of the developed world, but an alarming threat to public health worldwide. Before the 20th Century, despite the warnings of empirical physicians like Galen and Hippocrates, many cultures have harbored the false belief that obesity was a sign of health [1]. However, robust medical evidence has dispelled this notion and revealed in detail how obese individuals are prone to a plethora of diseases. These include, most seriously: cardiovascular disease (CVD), hypertension, Type 2 diabetes mellitus (T2D) and the endocrine complications of metabolic syndrome. More specifically, our recently deepened

(C) 2010 Wolters Kluwer Health | Lippincott Williams \& Wilkins

Correspondence to Gerald V. Denis, Boston Nutrition Obesity Research Center; Cancer Research Center; Boston University School of Medicine, Boston, MA 02118, USA, Tel: +1 617414 1371; fax: +1 617638 5673; gdenis@bu.edu.

The views expressed here do not necessarily reflect those of the NIH or any other organization with which the authors are affiliated. Any errors or omissions are of course solely the responsibility of the authors. 
understanding of causation, not just correlation, has changed this field of research, demanding that new studies of adipose tissue intersect trends in the immunology, endocrinology and metabolism disciplines. As the worldwide prevalence of obesity soars, the need is urgent for a much deeper biological understanding of the mechanisms that link obesity and T2D, in order to design new drugs and therapies to stave off the impending crisis in public health.

Although the innate immune system evolved to provide humans with important sensing mechanisms to defend against microbial pathogens, certain undesirable effects of this sensitivity were not felt as long as calorie intake remained low and physical activity was maintained. However, within the last several decades, the prevalence of high-calorie diets has increased worldwide, built on a human taste preference for sugary beverages and fatty foods. Increased sedentary patterns of activity as a result of a dramatically expanding information economy and increasing automation of industrial and agricultural production, widely available and inexpensive 'fast foods' and stressful urban lifestyles, have combined to create a disastrous, worldwide public health crisis of obesity and its comorbidities, including CVD and T2D.

The white adipose tissue (WAT) of vertebrates was regarded for many years to be an inert, passive reservoir for storage of excess energy. However, WAT has recently shown also to be a tissue of many talents. It is now clear that WAT also plays a major role not only in selfregulation, but also in endocrine control of processes that affect the whole organism. First, it functions as a lever, controlling energy expenditure and feeding behavior. Secondly, the adipocytes that form the WAT engage in mutual regulation with the innate immune system. Interestingly, certain functional relationships between the insect 'fat body', which is functionally similar to mammalian WAT and liver and the innate immune system are conserved among Drosophila, mice and humans [2]. This relationship suggests a deep evolutionary history of crosstalk among these cellular lineages. Adipocytes are also a source of a broad variety of soluble factors, called 'adipokines', that form an intricate network of paracrine, autocrine and endocrine relationships between the adipocytes themselves; the stromal vascular fraction of WAT that contains fibroblasts, pericytes, preadipocytes; and, above all, immune cells such as macrophages, B and T cells and natural killer cells.

When performing its main function of energy storage, WAT usually stays metabolically balanced; however, when 'overworked' due to hypernutrition or other metabolic stresses, serious pathologies soon emerge. Upon challenge with excess calories, an early response of the human body is to expand adipose depots. The function of energy storage becomes demanding on adipocytes, such that they develop cellular stress when the load becomes overwhelming. The first trigger in the cascade of events that leads to an unhealthy, inflammatory state is still a point of debate. One view holds that an adipocyte-produced, proinflammatory set of cytokines recruits cells of the immune system into the WAT to create an inflamed depot. Another view suggests that certain resident cells of the immune system detect emerging adipocyte damage in fat and produce the first bolus of cytokines and chemokines that recruit macrophages to clear dead and dying adipocytes. WAT is a massive tissue relative to total body weight and is capable of producing a variety of bioactive molecules in significant amounts, thus even a small change in WAT function can have major whole-body consequences.

\section{Insulin resistance: the role of inflammation}

In addition to the metabolic insults experienced by the cardiovascular and endocrine systems, adipose tissue in obesity and states of obesity-driven insulin resistance typically must cope with a low-grade, chronic state of subclinical inflammation [3]. In different 
animal models of obesity and in obese patients, this state evinces elevated serum concentrations of C-reactive protein (CRP), Interleukin (IL)-6, IL-8 and tumor necrosis factor (TNF)- $\alpha$ [4]. One of the first consequences of elevated serum concentrations of these proinflammatory factors is a negative influence on the insulin-signaling pathway and decreased insulin sensitivity in many central and peripheral tissues, including WAT itself, which puts the obese patient firmly on the road to T2D. Although TNF- $\alpha$ was the first secreted adipokine shown to have functional importance for the emergence of insulin resistance, its pathogenic activity was soon found to be exacerbated by IL-6, IL-1 $\beta$, monocyte chemoattractant protein (MCP)-1, and several other factors. Even if regarded as acting mostly locally, the proinflammatory adipokines disperse beyond the borders of the WAT and promote insulin resistance and atherosclerosis elsewhere in the body. Although a comprehensive understanding of the genes and pathways that drive inflammation-associated insulin resistance awaits further research, the importance of proinflammatory cytokines in the emergence of insulin resistance first came to the attention of the field through the seminal work of Hotamisligil and Spiegelman [5]. The field has been very ably reviewed since then $[6,7,8 \bullet]$.

As discussed earlier, in most obese patients, the main effect of increased adiposity and adipocyte stress is a low-grade inflammation and its most probable outcome is insulin resistance; this pattern is widely observed in clinical populations. However, other outcomes are possible, in which WAT and metabolic dysfunction are uncoupled from BMI. For example, there is a cohort of patients, comprising up to about $25 \%$ of the adult obese population, who despite obesity, never develop T2D and severe dyslipidemia, which is a phenotype first identified by Ruderman et al. [9] and termed 'metabolically healthy but obese' (MHO). Conversely, another cohort of patients termed 'metabolically obese but normal weight' (MONW), develop this low-grade inflammatory state without becoming obese and extending the load on their WAT. The MHO individuals are at reduced risk for CVD and T2D [9-11], and significantly, studies have shown that they have a reduced inflammatory profile. Factors that couple obesity to insulin resistance are of great medical interest, because they underlie the etiology of obesity-driven T2D. Thus, the MHO individual is likely to provide a goldmine of information. The study of this population for genes and pathways that couple obesity to insulin resistance has the potential to identify novel, 'druggable' targets to help unhealthy obese patients avoid the worst comorbidities of their condition.

\section{Mechanisms of adipose tissue inflammation in obesity}

It has been widely noted that food intake, energy expenditure and fat depot activity are regulated by one of the most tightly controlled switches in the body, where even a slight deviation from caloric input/usage causes either failure to maintain body functions or a vast increase of storage load. It is believed that the threat of starvation biased this system toward the preference of extra consumption; unfortunately, similar evolutionary mechanisms seem to have shaped the inflammatory response in fat. WAT can easily become a source of large amounts of active proinflammatory molecules, because it is very responsive to inflammatory signals. It follows from this point that there could be a direct link between other chronic inflammatory diseases and severity of obesity complications. A number of studies now report a connection between chronic inflammatory conditions, such as rheumatoid arthritis and periodontal disease, and morbidity associated with metabolic syndrome. Pre-existing inflammatory conditions such as asthma [12•] and periodontal disease [13] thus can contribute to the development of insulin resistance as a major comorbidity in such patients. 


\section{Role of adipose tissue macrophages}

As discussed earlier, WAT in obesity undergoes several deleterious changes, including expansion of extracellular matrix; adipocyte stress, hypertrophy and cell death [14]; and elevated production of proinflammatory adipokines that recruit immune cells [6,15-17]. These cells promote development of the chronic inflammatory state primarily attributable to adipose tissue macrophage (ATM) function [18-20]. The responses of the recruited macrophage population to the WAT are complex. Specifically, macrophages infiltrate WAT as circulating monocytes in response to WAT secretion of MCP-1, which recruits monocytes that express the $\mathrm{C}-\mathrm{C}$ chemokine receptor $(\mathrm{CCR}) 2[18,19,21]$. These proinflammatory $\mathrm{F} 4 / 80^{+} \mathrm{CD} 11 \mathrm{~b}^{+} \mathrm{CD} 11 \mathrm{c}^{+}$macrophages are directly implicated in WAT inflammation and the development of insulin resistance [22,23]. In support of this point, deletion of the CD11c compartment protects whole-body insulin sensitivity and glucose tolerance in mice [24]. Consistent with this mechanism, it has recently been reported that the circulating monocytes of obese insulin resistance and T2D patients display significantly elevated markers of this proinflammatory phenotype [25].

In obesity, production of TNF- $\alpha$ in liver, fat and muscle by infiltrating, proinflammatory ('M1 polarized') ATMs is clearly established to promote insulin resistance [5]. These 'classically activated' macrophages can be M1-polarized in vitro by exposure to bacterial endotoxin or cytokines that are secreted from Th1-polarized T cells, such as interferon- $\gamma$. High fat feeding promotes a macrophage phenotype switch within WAT to the M1 state [22]. The M1 state exists on a functionally defined spectrum with an anti-inflammatory ('M2 polarized') state. These 'alternatively activated' macrophages can be M2-polarized by exposure to cytokines that are secreted from Th2-polarized T cells, such as IL-4 and IL-13.

Adipocyte death in mouse models of diet-induced obesity (DIO) correlates with WAT inflammation, an increased M1/M2 ratio and the development of whole-body insulin resistance [26]. The CD11 $\mathrm{c}^{+}$ATM populations are also involved in scavenging dead adipocytes (in 'crown-like structures', histologically defined by CD11c staining of myeloid cells that cluster around dead or dying adipocytes in WAT sections) [14] and transiently remodeling the WAT, which then exhibits activities connected with M2-associated genes, such as arginase, IL-10, IL-4 and TGF- $\beta$ [27]. Relatively high expression of M1 cytokines is associated with metabolic complications of obesity, including insulin resistance, in both rodent models and humans. The net balance of these M1 and M2 inputs is thought to define the profile and magnitude of adipose tissue inflammation.

However, an interesting new report from the Olefsky group [28•] has revealed a functional plasticity in ATMs that belies this straightforward model of M1 vs. M2 polarization. Upon reversal of high-fat diet and return to normal caloric intake in a mouse model system, glucose tolerance and insulin sensitivity improve, but numbers of $\mathrm{F} 4 / 80^{+} \mathrm{CD} 11 \mathrm{~b}^{+} \mathrm{CD} 11 \mathrm{c}^{+}$ macrophages that infiltrate epididymal fat (the most inflammatory depot in this model) remain elevated. Instead of expressing a proinflammatory transcriptional signature, this tissue expressed reduced transcripts of TNF- $\alpha$, IL-1 $\beta$, IL-6, IL-10 and IFN- $\gamma$. Sorted CD11 ${ }^{+}$ epididymal ATMs showed significantly reduced TNF- $\alpha$ and IL- $1 \beta$ production, suggesting that ATMs are capable of repolarization to an anti-inflammatory phenotype, despite CD11c expression. This mechanism is consistent with amelioration of metabolic parameters in patients after various weight loss-focused dietary interventions (for review, see ref. [29]) and with rapid improvement observed in obese, nondiabetic patients after bariatric surgery [30]. ATM populations therefore likely respond to local metabolic microenvironment with a reduced inflammatory phenotype that bodies well for obese patients before $\beta$ cell failure and the emergence of insulin-dependent diabetes. 


\section{Adipokines and T cells}

$\mathrm{T}$ cells are also recruited to WAT in DIO through 'regulated on normal T cell expressed and secreted' (RANTES/CCL5) and its receptor CCR5 in adipose tissue [31,32], where their Th1/Th2 polarization and proliferation are influenced by macrophage-produced cytokines [33]. T cells also play a major role in insulin resistance [34] through macrophage recruitment [35] and Th17 (proinflammatory) [36] vs. T regulatory (Treg; antiinflammatory) [37•] polarization in WAT. Although ATMs infiltrate WAT during obesity and are an important source of proinflammatory cytokines thought to drive insulin resistance $[3,5,18,19]$, it is likely that not only are the ATMs using cytokines to communicate with adipocytes, but also the adipocytes are using adipokines to communicate with ATMs and T cells. There are a number of points at which this mechanism could provide a measure of protection against insulin resistance. Given their reduced inflammatory profile, some of these factors may be operative in MHO individuals [10,11,38,39].

\section{Recent findings}

No matter how complex the multifactorial network behind complications of obesity, its outputs converge on some definable proinflammatory and anti-inflammatory switches. For instance, one such potential balance point in MHO patients could be levels of adiponectin, an anti-inflammatory adipokine that is secreted by WAT; adiponectin levels drop in T2D patients. Adiponectin is known as a potent insulin sensitizer; high levels of adiponectin predict a dramatically lower myocardial infarction rate in men [40]. Likewise, it is possible that, among the forces that comprise the lever between 'good' and 'bad' fat deposition, some may be crucial and sufficient for successful therapeutic manipulation, such as peroxisome proliferator-activated receptor $\gamma$ (PPAR $\gamma$ ). PPAR $\gamma$ agonists and modulators are currently being tested in a vast array of clinical trials (for review, see ref. [41]). One of the keys to the mechanism of their action may be the newly revealed Brd2 pathway. Mice that exhibit reduced whole-body expression of the gene $\mathrm{Brd} 2$ uncouple obesity from insulin resistance [42••]. The WAT of these animals is dramatically expanded on normal chow supplied $a d$ libitum, rather than high-fat diet or forced feeding, yet WAT remains healthy and lacking in inflammatory infiltrates. After 12 months, adult male mice weigh about $90 \mathrm{~g}$, which is roughly equivalent to $180 \mathrm{~kg}$ (BMI $\sim 50)$ for adult male humans. Yet these animals never develop T2D; throughout their lives they are able to clear glucose better than matched wild type mice. Furthermore, low Brd2 levels resemble the action of thiazolidinedione drugs like rosiglitazone, which are used to sensitize obese patients to insulin action. PPAR $\gamma$-dependent transcription is greatly induced under ' $b r d 2 l o$ ' conditions, leading to enhanced adipogenesis $[42 \bullet \bullet$.

This discovery provides an exciting opportunity to investigate Brd 2 function in inflammatory cells and adipocytes of obese patients, to test the hypothesis that MHO patients are protected from T2D in part because of reduced Brd2 levels. Whether or not the ' $b r d 2 l o$ ' mouse proves to be a superb model for MHO patients, it presents an excellent example of how a single intervention at the basic transcriptional level influences such an intricate system as the inflammatory state of the fat tissue and whole body insulin sensitivity. Such mechanisms would have strong explanatory power to stratify obese patients into high and low-risk cohorts, and may offer a totally novel, 'druggable' therapeutic pathway for the high-risk patients.

In humans, $B R D 2$ is located at locus 21.3 on chromosome $6 \mathrm{p}$, within the class II major histocompatibility complex (MHC), flanked by genes that are involved in antigen processing. The $B R D 2$ sequence encodes a transcriptional coactivator [43-45] and bears no resemblance to any of the nearby genes in the MHC. Consistent with the inflammatory mechanism, a very recent report has identified human SNPs in the BRD2 locus that are 
significantly associated with rheumatoid arthritis, which is driven by autoimmune and inflammatory processes [46]. Links between Type 1 diabetes and alleles of the human class II MHC are well established [47]. Specifically, these include genes that regulate autoimmune processes. Other genes at $6 \mathrm{p} 21.3$, such as tumor necrosis factor $(T N F)$, are linked to inflammation and T2D in the context of obesity. Additional study of the relationships between genes that regulate responses of the innate immune system, such as the TLR2 and TLR4 pathways, NFKB and inflammation in the context of obesity, are likely to yield a wealth of insights into mechanisms that couple obesity to T2D and its comorbidities, and identify novel targets for therapy.

\section{Conclusion}

The present review discusses the functional interconnections between WAT and inflammatory cells in the context of diet-induced obesity in humans and animal models. Current research has established links between innate immune responses, chronic inflammation and the comorbidities of obesity, such as T2D and CVD. These links provide a rationale for a hypothesis that mild immunodeficiency might protect some obese individuals from these risks, whereas inflammatory hypersensitivity might increase the risks in others. This hypothesis receives support from the strong epidemiological correlation between asthma and insulin resistance in obese patients, for example, and raises the possibility of new biomarkers for high-risk groups.

Additional research is urgently necessary to identify the functional links between adipose cells and the genes of the innate immune system that control inflammatory signal transduction, immune cell infiltration into adipose tissue, macrophage and T-cell proliferation and survival, and adipocyte/immune cell crosstalk through cytokine and adipokine signaling, in order to understand and to block the cascade of chronic inflammation that leads to insulin resistance. Genes of the MHC, such as BRD2 and TNF; genes that control innate immune sensing, such as TLR2 and TLR4; genes that regulate inflammatory signal transduction pathways, such as IRAK and NFKBI; and genes that control adipocyte fate and function, such as $P P A R G$; likely interact in the pathogenesis of human insulin resistance in this context. Furthermore, the role of chromatin and transcriptional coactivation/corepression of obesity-relevant target genes, including epigenetic mechanisms that regulate signal transduction in obesity, demands much deeper study, as shown by the surprising coactivator/corepressor activity of the Brd2 protein.

In summary, it is clear that the innate immune system served an important role during the early millennia of human development, but modern society is vulnerable to the unanticipated, negative effects of these pathways, made apparent by the consequences of high BMIs that few humans had previously experienced, but that now are alarmingly common.

\section{Acknowledgments}

We thank Kathy Strissel, Fangnian Wang and other members of the Boston Nutrition Obesity Research Center (BNORC), particularly Caroline Apovian, Barbara Corkey, Steve Farmer, Susan Fried, Andrew Greenberg, Martin Obin, Paul Pilch and Neil Ruderman for valuable comments, discussion and insight. This work is supported in part by grants from the National Institutes of Health (NCI and NIDDK). BNORC is supported by NIDDK (P30 DK057521).

\section{References and recommended reading}

Papers of particular interest, published within the annual period of review, have been highlighted as: 
- of special interest

$\bullet$ of outstanding interest

Additional references related to this topic can also be found in the Current World Literature section in this issue (pp. 490-491).

1. Haslam D. Obesity: a medical history. Obesity Rev. 2007; 8:31-36.

2. Leclerc V, Reichhart JM. The immune response of Drosophila melanogaster. Immunol Rev. 2004; 198:59-71. [PubMed: 15199954]

3. Bastard JP, Maachi M, Lagathu C, et al. Recent advances in the relationship between obesity, inflammation, and insulin resistance. Eur Cytokine Netw. 2006; 17:4-12. [PubMed: 16613757]

4. Kahn SE, Zinman B, Haffner SM, et al. Obesity is a major determinant of the association of Creactive protein levels and the metabolic syndrome in type 2 diabetes. Diabetes. 2006; 55:23572364. [PubMed: 16873701]

5. Hotamisligil GS, Shargill NS, Spiegelman BM. Adipose expression of tumor necrosis factor- $\alpha$ : direct role in obesity-linked insulin resistance. Science. 1993; 259:87-91. [PubMed: 7678183]

6. Hotamisligil GS. Inflammation and metabolic disorders. Nature. 2006; 444:860-867. [PubMed: 17167474]

7. Hotamisligil GS, Erbay E. Nutrient sensing and inflammation in metabolic diseases. Nat Rev Immunol. 2008; 8:923-934. [PubMed: 19029988]

8. Hotamisligil GS. Endoplasmic reticulum stress and the inflammatory basis of metabolic disease. Cell. 2010; 140:900-917. [PubMed: 20303879] . This article highlights the importance of the 'unfolded protein response' of the endoplasmic reticulum as a crucial functional link between inflammation and obesity.

9. Ruderman NB, Schneider SH, Berchtold P. The "metabolically-obese," normal-weight individual. Am J Clin Nutr. 1981; 34:1617-1621. [PubMed: 7270486]

10. Wildman RP, Muntner P, Reynolds K, et al. The obese without cardiometabolic risk factor clustering and the normal weight with cardiometabolic risk factor clustering: prevalence and correlates of 2 phenotypes among the US population (NHANES 1999-2004). Arch Intern Med. 2008; 168:1617-1624. [PubMed: 18695075]

11. Succurro E, Marini MA, Frontoni S, et al. Insulin secretion in metabolically obese, but normal weight, and in metabolically healthy but obese individuals. Obesity (Silver Spring). 2008; 16:1881-1886. [PubMed: 18551117]

12. Shore SA. Obesity, airway hyperresponsiveness, and inflammation. J Appl Physiol. 2010; 108:735-743. [PubMed: 19875711]. This article emphasizes the growing importance of a systemic understanding of cytokine mediators of chronic inflammation and their crosstalk with adipocytes.

13. Mealey BL, Rose LF. Diabetes mellitus and inflammatory periodontal diseases. Curr Opin Endocrinol Diabetes Obes. 2008; 15:135-141. [PubMed: 18316948]

14. Cinti S, Mitchell G, Barbatelli G, et al. Adipocyte death defines macrophage localization and function in adipose tissue of obese mice and humans. J Lipid Res. 2005; 46:2347-2355. [PubMed: 16150820]

15. Schenk S, Saberi M, Olefsky JM. Insulin sensitivity: modulation by nutrients and inflammation. J Clin Invest. 2008; 118:2992-3002. [PubMed: 18769626]

16. Nishimura S, Manabe I, Nagasaki M, et al. Adipogenesis in obesity requires close interplay between differentiating adipocytes, stromal cells, and blood vessels. Diabetes. 2007; 56:15171526. [PubMed: 17389330]

17. Nishimura S, Manabe I, Nagasaki M, et al. In vivo imaging in mice reveals local cell dynamics and inflammation in obese adipose tissue. J Clin Invest. 2008; 118:710-721. [PubMed: 18202748]

18. Weisberg SP, McCann D, Desai M, et al. Obesity is associated with macrophage accumulation in adipose tissue. J Clin Invest. 2003; 112:1796-1808. [PubMed: 14679176] 
19. Xu H, Barnes GT, Yang Q, et al. Chronic inflammation in fat plays a crucial role in the development of obesity-related insulin resistance. J Clin Invest. 2003; 112:1821-1830. [PubMed: 14679177]

20. Clement K, Viguerie N, Poitou C, et al. Weight loss regulates inflammation-related genes in white adipose tissue of obese subjects. FASEB J. 2004; 18:1657-1669. [PubMed: 15522911]

21. Kanda H, Tateya S, Tamori Y, et al. MCP-1 contributes to macrophage infiltration into adipose tissue, insulin resistance, and hepatic steatosis in obesity. J Clin Invest. 2006; 116:1494-1505. [PubMed: 16691291]

22. Lumeng CN, Bodzin JL, Saltiel AR. Obesity induces a phenotypic switch in adipose tissue macrophage polarization. J Clin Invest. 2007; 117:175-184. [PubMed: 17200717]

23. Westcott DJ, Delproposto JB, Geletka LM, et al. MGL1 promotes adipose tissue inflammation and insulin resistance by regulating 7/4hi monocytes in obesity. J Exp Med. 2009; 206:3143-3156. [PubMed: 19995956]

24. Patsouris D, Li PP, Thapar D, et al. Ablation of CD11c-positive cells normalizes insulin sensitivity in obese insulin resistant animals. Cell Metab. 2008; 8:301-309. [PubMed: 18840360]

25. Satoh N, Shimatsu A, Himeno A, et al. Unbalanced M1/M2 phenotype of peripheral blood monocytes in obese diabetic patients: effect of pioglitazone. Diabetes Care. 2010; 33:e7. [PubMed: 20040670]

26. Strissel KJ, Stancheva Z, Miyoshi H, et al. Adipocyte death, adipose tissue remodeling, and obesity complications. Diabetes. 2007; 56:2910-2918. [PubMed: 17848624]

27. Duffield J. The inflammatory macrophage: a story of Jekyll and Hyde. Clin Sci (Lond). 2003; 104:27-28. [PubMed: 12519085]

28. Li P, Lu M, Nguyen MT, et al. Functional heterogeneity of CD11c-positive adipose tissue macrophages in diet-induced obese mice. J Biol Chem. 2010; 285:15333-15345. [PubMed: 20308074] . This article highlights an interesting, new 'phenotype switch' mechanism of inflammatory macrophages that infiltrate adipose tissue, which has important and encouraging implications for the possibility that straightforward dietary adjustments can quickly improve metabolic parameters in obese patients through rapid responses of the adipose tissue macrophages.

29. Klimcakova E, Kovacikova M, Stich V, Langin D. Adipokines and dietary interventions in human obesity. Obesity Rev. 2010; 11:446-456.

30. Stubbs RS, Wickremesekera SK. Insulin resistance in the severely obese and links with metabolic co-morbidities. Obes Surg. 2002; 12:343-348. [PubMed: 12082884]

31. Wu H, Ghosh S, Perrard XD, et al. T-cell accumulation and regulated on activation, normal T cell expressed and secreted upregulation in adipose tissue in obesity. Circulation. 2007; 115:10291038. [PubMed: 17296858]

32. Surmi BK, Hasty AH. The role of chemokines in recruitment of immune cells to the artery wall and adipose tissue. Vascul Pharmacol. 2010; 52:27-36. [PubMed: 20026286]

33. Loke P, MacDonald AS, Robb A, et al. Alternatively activated macrophages induced by nematode infection inhibit proliferation via cell-to-cell contact. Eur J Immunol. 2000; 30:2669-2678. [PubMed: 11009101]

34. Kintscher U, Hartge M, Hess K, et al. T-lymphocyte infiltration in visceral adipose tissue: a primary event in adipose tissue inflammation and the development of obesity-mediated insulin resistance. Arterioscler Thromb Vasc Biol. 2008; 28:1304-1310. [PubMed: 18420999]

35. Nishimura S, Manabe I, Nagasaki M, et al. CD8+ effector T cells contribute to macrophage recruitment and adipose tissue inflammation in obesity. Nat Med. 2009; 15:914-920. [PubMed: 19633658]

36. Winer S, Paltser G, Chan Y, et al. Obesity predisposes to Th17 bias. Eur J Immunol. 2009; 39:2629-2635. [PubMed: 19662632]

37. Feuerer M, Herrero L, Cipolletta D, et al. Lean, but not obese, fat is enriched for a unique population of regulatory T cells that affect metabolic parameters. Nat Med. 2009; 15:930-939. [PubMed: 19633656]. This article highlights pathbreaking new work on the role of regulatory $T$ cells (Tregs) in adipose tissue and brings attention to the previously unappreciated potential of Tregs to improve metabolic function in human obesity and animal models of obesity. 
38. Bonora E, Willeit J, Kiechl S, et al. U-shaped and J-shaped relationships between serum insulin and coronary heart disease in the general population. The Bruneck Study. Diabetes Care. 1998; 21:221-230. [PubMed: 9539986]

39. Karelis AD, Faraj M, Bastard JP, et al. The metabolically healthy but obese individual presents a favorable inflammation profile. J Clin Endocrinol Metab. 2005; 90:4145-4150. [PubMed: 15855252]

40. Pischon T, Girman CJ, Hotamisligil GS, et al. Plasma adiponectin levels and risk of myocardial infarction in men. JAMA. 2004; 291:1730-1737. [PubMed: 15082700]

41. Blackburn GL. From bench to bedside: novel mechanisms and therapeutic advances through the development of selective peroxisome proliferator-activated receptor gamma modulators. Am J Clin Nutr. 2010; 91:251S-253S. [PubMed: 19923371]

42. Wang F, Liu H, Blanton WP, et al. Brd2 disruption in mice causes severe obesity without type 2 diabetes. Biochem J. 2010; 425:71-83. [PubMed: 19883376]. This article announces a completely unexpected and potentially revolutionary finding that involves a new gene called $\mathrm{Brd} 2$, which encodes a transcription co-factor not previously shown to be important for energy balance. Wholebody reduction in $\mathrm{Brd} 2$ levels renders mice exceptionally obese while preserving their insulin sensitivity. Because humans share this pathway, this report has significant diagnostic and therapeutic implications for the role of BRD2 in 'metabolically healthy obese' humans. This molecular pathway likely influences human sensitivity to the inflammation-based diseases that obesity can trigger.

43. Denis GV, Green MR. A novel, mitogen-activated nuclear kinase is related to a Drosophila developmental regulator. Genes Dev. 1996; 10:261-271. [PubMed: 8595877]

44. Sinha A, Faller DV, Denis GV. Bromodomain analysis of Brd2-dependent transcriptional activation of cyclin A. Biochem J. 2005; 387:257-269. [PubMed: 15548137]

45. Denis GV, McComb ME, Faller DV, et al. Identification of transcription complexes that contain the dual bromodomain protein Brd2 and chromatin remodeling machines. J Proteome Res. 2006; 5:502-511. [PubMed: 16512664]

46. Mahdi H, Fisher BA, Källberg H, et al. Specific interaction between genotype, smoking and autoimmunity to citrullinated alpha-enolase in the etiology of rheumatoid arthritis. Nat Genet. 2009; 41:1319-1324. [PubMed: 19898480]

47. Leslie RD. Predicting adult-onset autoimmune diabetes: clarity from complexity. Diabetes. 2010; 59:330-331. [PubMed: 20103711] 